\title{
Méthode pour un plan à moyen terme
}

\author{
M. FALKOWSKI*
}

\section{RÉSUMÉ}

L'université de Genève dispose d'un système original de planification à moyen terme sous forme des plans de développement quadriennaux. La responsabiblité de ces plans, inscrits dans la Loi sur l'Université du 26 mai 1973, incombe à la Commission de développement (C.D.), organe consultatif permanent du Rectorat.

La Commission de développement est composée des représentants des facultés et écoles et est présidée par un vice-recteur qui dirige également le Service de développement (S.D. trois collaborateurs scientifiques et le secrétariat) appartenant à l'administration centrale, chargé d'études, d'élaboration du plan et de la recherche institutionnelle.

Le rôle de la Commission de développement est spécifique et ne se limite pas à la planification seulement. La C.D. est un lieu privilégié de la réflexion prospective de l'Université et de ce fait, les aspects qualitatifs de développement sont constamment confrontés aux aspects quantitatifs. Ainsi, le terme "développement" recouvre un vaste champ d'investigation et ne s'identifie pas à une simple technique de planification. Par cette approche également, deux autres buts sont visés: d'une part, l'harmonisation des structures academiques et administratives, chose particulièrement importante dans une université européenne typique et, d'autre part, la liaison entre la planification a moyen terme et celle à long terme (prévision du nombre d'étudiants, des locaux, etc.)

L'article se réfère à l'expérience de la planification à l'Université de Genève et se base en grande partie sur le chapitre "Méthode" du deuxième plan de développement pour les années 1977-1980.

\footnotetext{
ABSTRACT

The University of Geneva has at its disposal an innovative system of medium range planning in the form of 4-year development plans. These plans, written into the "Loi sur l'Universite" (the University Act) and dated May 26, 1973, are administered by the Commission de développement (C.D.) (Development Commission), a standing advisory body to the vice-chancellor's office.

The C.D. is made up of representatives of faculties and schools and is chaired by a vice-chancellor who also heads the Service de développement (S.D.)/Development Service) - a team of three researchers and their secretarial office. The S.D. is under the 
central administration and is responsible for studies, development of the plan and institutional research.

The role of the C.D. is specific and is not limited only to planning. The C.D. lends itself well to university forecasting and, because of this, the qualitative and quantitative aspects of development are constantly compared. Thus, the term "development" covers a wide field of investigation and is not identified with a mere planning technique. Through this approach, two other ends are also sought: on the one hand, bringing academic structures into line with administrative ones. (an extremely important aspect in a typical European university) and. on the other hand, coordinating medium and long range planning (forecasting the number of students, space etc.)

The article refers to the planning experience of the University of Geneva and is based. in the main, on the chapter entitled "Methode" of the second development plan for the years 1977-1980.

\section{Remarques préliminaires}

La planification à moyen terme peut jouer un double rôle: à l'intérieur de l'université, elle peut contribuer à mieux définir et hiérarchiser les objectifs de l'enseignement et de la recherche, et faciliter la gestion des ressources humaines et financières. Vis-à-vis de l'extérieur, le Plan pourrait être un instrument d'information. Ce double but assigné au plan doit trouver son expression dans la forme de sa publication transparente, lisible et quelque peu même didactique.

Quant au choix des méthodes, il devrait être opéré en fonction des moyens disponibles, et dicté par le souci d'éviter que le plan ne devienne inutilement une sorte de contrainte institutionnelle supplémentaire. Deux approches pourraient être envisagées:

- la construction d'un modèle formé d'ensemble d'équations mathématiques auxquelles correspondrait une planification directive;

- une planification plus pragmatique se contentant d'optimatisation des choix partiels.

L'expérience de certaines universités prouve que la construction de modèles théoriques de développement est possible. Les travaux dans ce domaine se poursuivent d'ailleurs activement. La difficulté réside moins sur le plan théorique que sur celui de l'application de ces modèles, qui s'avèrent souvent trop abstraits pour être utiles dans la gestion courante des universités*.

Les auteurs ont renoncé à mettre sur pied une planification directive fondée sur un modèle de développement complet de l'ensemble de l'université, en optant pour la formule plus souple de la planification indicative. L'approche plus pragmatique qui a été choisie entraîne cependant aussi un certain nombre de problèmes, parmi lesquels le plus important est peut-être qu'il faille prendre des décisions sans être en mesure, par manque d'un modèle théorique, d'en prévoir l'ensemble des conséquences.

La planification pragmatique et indicative est - selon cette conception - , un outil dynamique et évolutif qui doit être constamment perfectionné et mis à jour à mesure des expériences faites tant au niveau pratique que théorique. Cette mise à jour et ce perfection-

* Voir les travaux du Centre pour la recherche et l'innovation dans l'enseignement CERI/OCDE. 
nement demandent impérativement la participation active de tous les responsables de l'organisation universitaire.

\section{Contraintes}

Elles sont d'ordre méthodologique, institutionnel et subjectif.

Il est juste de rappeler que le premier Plan quadriennal à l'Université de Genève (19711975) avait le caractère d'une étude prospective basée principalement sur les prévisions du nombre d'étudiants et les données financières. Son but était surtout de situer les facultés les unes par rapport aux autres et, à un moindre degré, d'élaborer une politique différenciée de développement par une réflexion qualitative qui définissait un ensemble cohérent d'axes de développement. En cela même, sa portée méthodologique était limitée.

La tâche des auteurs du deuxième Plan quadriennal était déjà plus complexe. Il s'agissait de passer d'une prédiction non contraignante à la formulation d'un programme de développement en termes à la fois qualitatifs et quantitatifs, et d'estimer les moyens nécessaires à sa réalisation. Il fallut d'abord créer une base conceptuelle puis déterminer des procédures d'arbitrage, pour passer enfin à l'élaboration du plan. La méthodologie du deuxième plan présente cette particularité qu'elle est née avec le plan lui-même.

La contrainte institutionnelle pourrait se définir par le fait que la méthodologie et le plan devaient être formulés en l'absence d'une planification à moyen ou long terme qui, au niveau de l'Etat de Genève, situerait les problèmes de l'enseignement universitaire dans l'ensemble des orientations cantonales. D'un autre côté, les travaux sur la planification universitaire à l'échelle de la Confédération n'en sont qu'à leurs débuts.

Aux difficultés institutionnelles venaient s'en ajouter d'autres, de caractère subjectif, qui tenaient au manque d'une information statistique sur les divers aspects de l'enseignement et de la recherche dans les facultés, information indispensable pour formuler les options et les projets du plan. Il a été nécessaire d'entreprendre des études, de réunir une banque de données, de les élaborer et de les interpréter. Cette phase des travaux prêparatoires fut longue et onéreuse. Une certaine suscipicion des membres de la communauté universitaire à l'égard de la planification est toujours liée à l'expérience propre des parties impliquées. Or, c'est précisément cette expérience qui manquait tant au niveau de l'université qu'à celui des facultés et de leurs subdivisions.

Il faut remarquer que de nouvelles contraintes d'ordre conjoncturel et financier, liées à la récession, sont apparues au cours de l'élaboration du plan, remettant constamment en question les paramètres de la méthode.

\section{Buts de la planification}

Toute planification nécessite une définition explicite des buts à atteindre, en général sous une forme quantifiée et mesurable. Pour l'enseignement, et l'université en particulier, la planification s'avère plus complexe qu'en d'autres domaines, les objectifs, telle la qualité de l'enseignement et de la recherche, étant plus difficiles à quantifier. De surcroît, la diversité des facultés et des matières fait que la définition des objectifs ne saurait être un acte unique, réalisé spontanément, en une seule fois, mais le fruit de recherches menées à tous les niveaux: départements, facultés et écoles, Commission de développement et Rectorat. La synthèse de cette recherche relève de la Commission de développement, qui 
veille tout particulièrement à la coordination entre les projets généraux de l'université et les projets des facultés et écoles.

Les objectifs fixés dans le plan dépendent de deux catégories de facteurs:

- les facteurs qualitatifs, tels que les options socio-culturelles, le progrès de la science, la coordination des aspects relatifs à l'organisation de l'enseignement et de la recherche;

- les facteurs quantitatifs, comme les données conjoncturelles, la prévision du nombre d'étudiants, du personnel enseignant et administratif, la surface des locaux, certains aspects quantitatifs de l'enseignement (par exemple le taux d'encadrement).

L'intégration, au niveau de la méthode, des aspects qualitatifs et quantitatifs, semble primordiale, et la condition même de l'approche de l'enseignement, et de l'université en particulier. Pour la planification, le principe de non séparation entre le "qualitatif" et le "quantitatif" a donc un sens épistémologique fondamental.

L'élaboration du plan à partir de l'ensemble de ces facteurs pourrait:

- contribuer à une meilleure connaissance de la situation interne de l'université (pôles de développement, centres de gravité, direction de recherche, etc.);

- favoriser la coordination des nouveaux projets de développement au sein des facultés et au niveau interfacultaire, pour éviter leur double emploi;

- engendrer une politique concertée en matière d'équipement scientifique;

- permettre, en cas de difficultés financières, une procédure d'arbitrage de qualité entre les projets à tous les niveaux.

La définition des objectifs a, dans une large mesure, suivi le principe de participation à tous les échelons des structures universitaires. Les objectifs à atteindre dans l'enseignement, la recherche et les autres services, furent formulés par les départements, sections et facultés, d'abord en termes qualitatifs, puis en termes de postes, crédits de fonctionnement, équipement, etc. Ils furent articulés sur deux plans: celui de l'université et celui des facultés. Pour ce qui est de l'université dans son ensemble, le plan a repris les orientations et les options à moyen terme formulées par le Rectorat, dont les deux idées motrices sont le développement prioritaire des sciences humaines et l'ouverture de l'université vers l'extérieur. Les facultés ont, quant à elles, créé des groupes de travail chargés de l'élaboration d'une politique de développement pour l'enseignement et la recherche dans leurs subdivisions respectives.

\section{Paramètres du plan}

Il fut admis, dès le début des travaux, que le plan devait contenir des éléments qualitatifs et quantitatifs qu'il s'agissait d'intégrer dans un tout. Cette approche impliquait que l'on définit les priorités au niveau de l'université et de ses subdivisions, et écartait l'idée que le plan füt un simple instrument de ventilation de moyens financiers.

La méthode suivie fut celle des approximations successives, qui introduit deux paramètres principaux:

- les taux de croissance proposés aux facultés par la Commission de développement;

- les projets d'innovation adressés par les facultés sous la forme de demandes d'inclusion dans le plan.

Pour le taux de croissance, trois hypothèses (maximale, moyenne, minimale) ont été retenues pour chaque faculté. Elles tenaient compte d'une part, des limites de croissance 
possible et, d'autre part, d'une première répartition des moyens en fonction des prévisions du nombre d'étudiants et des taux d'encadrement.

Pour chaque hypothèse, la Commission de développement a fourni aux facultés le cadre quantitatif correspondant, appelé marge disponible.

Les facultés ont appliqué cette même procédure de répartition de la marge disponible jusqu'au niveau des unités budgétaires*.

Les projets d'utilisation de la marge disponible ont suivi un courant inverse. Les unités budgétaires formulaient leurs propositions et chaque projet devait faire l'objet d'une demande d'inclusion dans le plan, demande accompagnée de justificatifs qualitatifs et d'une information chiffrée détaillée. Ces indications fournies par les départements ou sections étaient ensuite transmises à la faculté qui, à son tour, regroupait les projets retenus , par ordre de priorité dans le cadre de la marge globale qui lui était attribuée.

Certains projets sortant du cadre donné et présentant une importance particulière pouvaient toutefois faire l'objet d'une demande spéciale, appelée hors budget. Les demandes hors budget devaient favoriser l'éclosion de projets novateurs, tels certains projets interfacultaires et interdisciplinaires, la création de nouveaux centres de gravité, des développements sectoriels urgents, etc.

La "demande d'inclusion dans le plan", qui était le principal outil de dialogue entre les départements, sections, facultés et la Commission de développement, a permis:

- de définir les conséquences des projets en ce qui concerne le personnel et l'équipement;

- de hiérarchiser les projets selon l'importance du sujet, en considérant l'ensemble des besoins de la subdivision, et de favoriser ainsi la mise en place d'éléments nécessaires à un programme qualitatif et quantitatif de développement de la subdivision à plus long terme;

- de préparer un lien de procédure entre le plan et les budgets annuels qui, dans l'esprit de la méthode utilisée, devaient de plus en plus exprimer un planning à court terme issu du plan pluriannuel, et se traduire en projets "connus" et "justifiés".

Dans cette approche méthodologique, on distingue donc entre la planification en tant que processus continu, le plan lui-même, expression momentanée de ce processus, et enfin l'évaluation permanente des écarts du plan dans le processus de gestion de l'université.

\section{Etapes de l'élaboration du plan}

Dans la première étape, la Commission et le Service de développement ont procédé à des études approfondies en vue de définir un certain nombre de données qualitatives et quantitatives de base et de dégager des critères de développement. Ces études concernaient:

- les prévisions du nombre d'étudiants par faculté et école pour la période 1975-1990;

- l'évolution du taux d'encadrement du personnel enseignant, administratif et technique;

- les "trends" antérieurs de développement des facultés;

- l'enquête systématique sur la part de la recherche et de l'enseignement dans les activités universitaires; et

- l'étude des problèmes généraux: organisation des études, diversification des voies d'entrée et de sortie, troncs communs, études à temps partiel, etc., ainsi que d'autres éléments de la politique universitaire cantonale et fédérale.

* C'est-à-dire les départements, regroupés à leur tour en sections et facultés. 
Dans la deuxième étape, et sur la base des études précédentes, il s'est agi de fixer le cadre quantitatif de développement probable pour l'ensemble de l'université et ses subdivisions. La détermination de la masse financière globale selon trois hypothèses de développement et selon les trois secteurs principaux (sciences, médecine, sciences humaines) fut accompagnée de la mise au point de la méthode de calcul de la marge de croissance (salaires standard, conversion des fractions de postes en postes à plein temps, etc.).

Sur le plan qualitatif, il y a lieu de mentionner les deux "prises de position de la Commission de développement" contenues dans l'enquête adressée à toutes les unités universitaires, concernant l'organisation des études et l'utilisation des nouvelles méthodes et techniques pédagogiques pour le perfectionnement de l'enseignement.

L'aboutissement de cette phase des travaux fut l'élaboration et le lancement de l'enquête pour l'établissement du plan, principal outil de travail de la Commission de développement.

Avec la récolte des réponses des facultés débutait, la troisième étape, celle de la consultation entre la Commission et le Service de développement d'une part, et les facultés, sections et départements d'autre part.

Les propositions chiffrées, accompagnées des justificatifs et présentées selon trois hypothèses de croissance, ont permis d'établir un ordre de priorité des besoins pour la plupart des facultés.

L'ensemble des réponses fut alors réparti en deux groupes: les "hors-budgets" et les "budgets ordinaires". Les "hors-budgets" constituaient une réserve déduite de la marge de croissance totale disponible, qui elle-même fut fixée à $12 \%$ du budget de l'année de référence (1975) pour les quatre années couvertes par le plan.

C'est à ce moment qu'une procédure d'arbitrage basée sur des critères de choix explicites fut appliquée.

On trouvera en annexe, une liste des critères définis par un groupe de travail de la Commission de développement. Ces critères furent pondérés selon l'importance qui leur avait été accordée pour le choix des projets. La première partie de la liste énumère les critères généraux appliqués seulement au niveau de l'arbitrage entre facultés. Ils servent à déterminer l'ẹnveloppe des crédits disponibles pour chaque faculté, dans le cadre des budgets normaux. Une fois ces masses déterminées, les critères pour l'enseignement et la recherche (que l'on trouve dans la seconde partie) permettent de hiérarchiser les projets selon les facultés, et à l'intérieur de l'enveloppe fixée. En effet, les facultés devraient pouvoir accorder l'hypothèse de croissance maximum à certaines unités, alors que d'autres ne bénéficieraient que du minimum ou même d'aucune croissance réelle dans les cas extrêmes.

Sur la base des dossiers fournis, le Service de développement entreprit, dans une quatrième étape, d'appliquer les critères, d'abord aux projets "hors-budget", puis pour la définition des enveloppes financières des facultés. Les résultats furent ensuite confrontés aux données d'un groupe réunissant, pour chaque faculté, quelques professeurs autour du délégué de la faculté à la Commission de développement. En commun, les critères furent revus un à un pour une première appréciation et sans, bien entendu, qu'il ait jamais été question d'une application rigide. La pratique montra cependant que dans l'ensemble les résultats concordaient et permettaient une bonne estimation de l'ordre de priorité des projets. Par ailleurs, cette procédure obligeait les membres du groupe de travail à examiner à fond tous les aspects des projets proposés. Ce résultat à lui seul justifierait la méthode utilisée. 
La collaboration du groupe de professeurs fut indispensable pour effectuer des coupures et réductions aux demandes, car il était impossible d'appliquer la même règle pour tous les cas. Certains projets furent abandonnés, d'autres fortement réduits et il s'agissait alors de procéder avec un maximum de discernement. De plus, il fallait adopter une attitude plus souple pour les cas particuliers où les critères s'appliquent mal.

Dans la cinquième étape, les résultats de l'arbitrage furent transmis à toutes les unités budgétaires pour information et mise au point définitive. A la suite des recours des facultés et écoles, des modifications durent être apportées, après consultation et négociation (parfois difficiles à ce stade) jusqu'à l'acceptation définitive du plan par les unités concernées.

Conformément aux termes de la Loi sur l'université, le Rectorat, le Collège des recteurs et doyens ainsi que le Département de l'Instruction Publique du Canton de Genève ont été consultés et ont pu émettre leurs recommandations dont il a été tenu compte dans la rédaction finale du document qu'il appartenait au Conseil de l'université d'approuver.

\section{Relation plan-budget}

La matérialisation du Plan quadriennal se fait par l'intermédiaire des budgets annuels. Le plan n'a donc de valeur que si le budget tient compte des priorités retenues pour la période quadriennale.

Cette liaison capitale plan-budget n'avait pas pu être réalisée pour la période du premier plan quadriennal. Ce fut, semble-t-il, la cause principale de sa rapide désuétude. Cette relation est donc aujourd'hui une des préoccupations fondamentales de la Commission de développement et de la Commission administrative (du budget).

La Commission de développement fut chargée d'étudier une procédure de liaison plan-budget qui soit permanente et officialisée. Cette procédure devra être très proche de celle qui amènera la révision périodique du plan.

Ainsi, pour la préparation du budget 1977 , la Commission administrative et la Commission de développement ont émis des directives précises, imposant aux facultés et autres subdivisions de tenir compte des priorités retenues par le plan. Ces directives devaient permettre l'introduction dans le budget de projets de développement selon la procédure suivante: chaque faculté était invitée à mentionner sur des formules ad hoc, en sus d'une mise à jour du budget 1977, un budget de développement qui tînt compte de deux ordres de priorités $A$ et $B$. La priorité $A$ ne devait pas dépasser les 3/5ème et la priorité $B$ les $2 / 5$ ème de la marge globale admise. Les demandes de développement s'écartant des propositions contenues dans le plan nécessitaient des justificatifs précis.

Cette procédure a permis de respecter l'orientation du deuxième plan quadriennal en retenant pour la présentation du budget 1977 la priorité A, qui tenait compte des demandes de développement considérées les plus urgentes.

\section{Révision du plan}

Il est évident que les options inscrites dans le plan peuvent être modifiées ou remplacées selon les changements de conditions et de besoins. La Commission de développement a cependant institutionnalisé un système de contrôle des écarts du plan et a établi des procédures en commun avec les unités intéressées. Cette procédure analyse d'une part le budget de l'année précédente par rapport au plan, d'autre part les demandes budgétaires courantes en fonction des options retenues à moyen terme. 
Liste des criteres de la procedure d'arbitrage*

Critères basés sur des données disponibles

a) Nombre d'étudiants: prévisions

b) Taux d'encadrement

c) Coordination romande

d) Centres de gravité de l'Université de Genève

e) Politique suisse de la science (basée sur le rapport du Conseil Suisse de la Science)

f) Fonds National de la Recherche Scientifique

Critères de politique générale à confronter avec les demandes ENSEIGNEMENT

a) Services interdisciplinaires et interfacultaires

b) Débouchés

c) Masse critique et formation d'approfondissement

d) Diversification des études:

- études courtes

- études à la carte et système d'unité de valeur

- perméabilité des voies d'études. Troncs communs

- nouveaux diplômes

e) Demandes sociales de formation

- enseignement secondaire

- environnement: organisations internationales

- clientèle spécifique

f) Education permanente

g) Innovation des méthodes et des techniques d'enseignement (perfectionnement pédagogique etc.)

h) Orientation universitaire et professionnelle

RECHERCHE

a) Potentiel d'innovation et production scientifique

b) Interdisciplinarité

c) Masse critique

d) Collaboration interuniversitaire et internationale

e) Nouveaux centres de recherche

f) Diffusion de la recherche

g) Initiation à la recherche

h) Demande sociale:

- environnement: organisations internationales

- clientèle spécifique

* sans pondération 